\section{Longevidad y revolución digital. Contribución de las tecnologías de la información y de la comunicación al envejecimiento saludable}

\author{
Longevity and digital revolution. \\ The contribution of information and \\ communication technologies to \\ healthy aging
}

Mónica Padial Espinosa ${ }^{1 ; *}$

Sandra Pinzón Pulido²

Juan Manuel Espinosa Almendro ${ }^{3}$

Alexandre Kalache ${ }^{4}$

Francisco Garrido Peña ${ }^{5}$

1. Máster en Actividad Física y Salud. Escuela Andaluza de Salud Pública. Granada. España.

2. Doctora en Ciencias de la Salud. Escuela Andaluza de Salud Pública. Granada. España.

3. Doctor en Medicina. Centro de Salud El Palo. Málaga. España.

4. Doctor. International Longevity Centre Brazil. Río de Janeiro. Brasil.

5. Doctor en Filosofía. Universidad de Jaén. Jaén. España.

*Autor para correspondencia.

Correo electrónico: monicapadial@gmail.com (Mónica Padial Espinosa).

Recibido el 15 de mayo de 2018; aceptado el 11 de noviembre de 2018

\section{RESUMEN}

Objetivo: Sintetizar el conocimiento disponible sobre la contribución de las tecnologías de la información y de la comunicación al envejecimiento activo y saludable. Metodología: En la primera fase de búsqueda se utilizó la base de datos GERION de la Biblioteca Virtual del Sistema Sanitario Público Andaluz (SSPA). En la segunda fase se realizó una búsqueda en Cochrane Library, PubMed, EMBASE, Medline, SciELO, MedlinePlus y ScienceDirect. Se utilizaron descriptores de lenguaje natural y lenguaje controlado. Se incluyeron estudios publicados en castellano e inglés en el período comprendido entre enero de 2013 y enero de 2018. Se realizó una metasíntesis cualitativa. Resultados: Se seleccionaron 262 estudios; se excluyeron 240: por estar duplicados, por no cumplir con los

criterios de inclusión, por no disponer del texto completo y por no guardar relación con el objeto de estudio. Se incluyeron 22 estudios en la síntesis cualitativa. A partir de la identificación de temas emergentes y su agrupación en categorías, se construyó un modelo conceptual que explica la relación entre envejecimiento activo y saludable e innovación tecnológica.

Conclusiones: Las tecnologías de la información y de la comunicación contribuyen al empoderamiento de las personas para el autocuidado; mejoran su autoeficacia y ayudan a mantener su autonomía en la toma de decisiones; favorecen su participación efectiva en los sistemas sanitarios y de cuidados, y contribuyen a la democratización de la salud. Su utilización está condicionada por el compromiso y la implicación de las personas mayores en su diseño, la adaptación a los entornos y preferencias de la persona usuaria, su simplicidad, comprensibilidad, utilidad percibida y facilidad de uso.

PALABRAS CLAVE: Envejecimiento, aplicaciones móviles, tecnología de la información.

\section{ABSTRACT}

Objective: synthesize the available knowledge on the contribution of information and communication technologies to active and healthy ageing. Methodology: GERION database from the Virtual Library of the Public Health System of Andalusia was used in the first search phase. In the second phase, a search was performed using Cochrane Library, PubMed, EMBASE, MEDLINE, SciELO, MedlinePlus, and Science Direct. Natural language and controlled language descriptors were used. Studies published in Spanish and English in the period January 2013 to January 2018 were included. Qualitative metasynthesis was carried out. Results: 262 studies were selected; 240 were excluded due to: duplication, not meeting the inclusion criteria, missing the full text, or not related to the object of study. 22 studies were included in the qualitative synthesis. From the identification of emerging issues and their grouping into categories, a conceptual model was constructed that explains the relationship between active and healthy ageing and technological innovation. Conclusions: Information and communication technologies contribute to the empowerment of people for self-care; improve their self-efficacy; help maintain their autonomy in decision-making; favor their effective participation in health and care systems; and contribute to the democratization of health. Its use is conditioned by the commitment and involvement of older people in its design, adaptation to the environments and preferences of the user, its simplicity, comprehensibility, perceived utility and ease of use.

KEYWORDS: Aging, elderly, informatics applications, health services for the aged.

\section{- INTRODUCCIÓN}

En las últimas décadas, la esperanza de vida se ha incrementado en promedio 30 ańos $^{1-3}$. Estos ańos extra de vida son un privilegio sin precedentes que se ha denominado la "revolución de la longevidad"2. Los años de vida ganados tienen importantes implicaciones derivadas principalmente del potencial in- cremento de personas mayores dependientes ${ }^{4}$. Sin embargo, una vida más larga también proporciona al individuo la oportunidad de invertir esos años de forma innovadora, tener más tiempo para la reflexión y las relaciones afectivas y sociales, y prestar mayor atención a la generatividad y a la genero$\operatorname{sidad}^{2,5}$. Así, la extensión de la vida puede generar bienestar para el individuo a la vez que desarrollo social y económico en el entorno en el que vives. 
La "revolución de la longevidad" coincide con otra tendencia global, la denominada "revolución tecnológica"'. Las tecnologías de la información y de la comunicación (TIC) han experimentado grandes avances en las últimas décadas, aportando soluciones a las necesidades de las personas en ámbitos diversos como la salud, los transportes, el ocio o el entretenimiento ${ }^{6}$. La informática actualmente disponible en cualquier ambiente permite cada vez más que los objetos se conecten a través de Internet y que los sistemas reconozcan el contexto y puedan procesar el conocimiento para generar nuevo conocimiento ${ }^{6,7}$. Características de las TIC tales como el tamaño y manejabilidad de los dispositivos móviles; la mejora continua en el alcance, la seguridad y la fiabilidad en las comunicaciones; el desarrollo de interfaces inteligentes; las técnicas de procesamiento de lenguaje natural; $y$, la inteligencia artificial entre otros, acercan cada vez más la tecnología a las personas ${ }^{6}$. Si bien en edades avanzadas muchas personas carecen de habilidades básicas para aprovechar los beneficios potenciales de las TIC y muestran menos interés que el resto de la población general, fomentar estas habilidades favorece su inclusión social ${ }^{8}$, mejora su calidad de vida y refuerza sus vínculos con la sociedad?.

Una tercera revolución está también en marcha. Se trata del "Big Data”, una revolución para los sistemas sanitarios en el mundo entero . Décadas de digitalización de registros clínicos empiezan a devolver a los sistemas y a los individuos conocimiento útil para la toma de decisiones en salud, la garantía de tratamiento oportuno y adecuado y el descubrimiento de nuevas terapias y enfoques para la provisión de cuidados, entre otros ${ }^{7}$.

Las TIC no solo prestan apoyo en la atención a la enfermedad y la discapacidad, sino que cada vez más ofrecen alternativas para el mantenimiento de la capacidad funcional y cognitiva de las personas a medida que envejecen ${ }^{1}$. En este contexto resulta imprescindible disponer de un modelo conceptual que permita comprender cuál es la contribución que las TIC proporcionan al envejecimiento saludable ${ }^{10}$. Este modelo conceptual será útil para el diseño de las soluciones tecnológicas que apoyan las intervenciones en envejecimiento saludable, así como para el establecimiento de indicadores de evaluación de resultados. Para ello, se propuso el siguiente estudio que, a través de una metasíntesis cualitativa, permitiera organizar el conocimiento disponible y construir el modelo conceptual.

\section{- MÉTODOS}

\section{Tipo de estudio}

Metasíntesis cualitativa como forma de revisión sistemática e integración de hallazgos de estudios primarios, cuyo propósito es crear una representación interpretativa, permaneciendo fiel a las interpretaciones de cada estudio particular. Bajo esta metodología, es posible sintetizar hallazgos de estudios cualitativos y cuantitativos ${ }^{11}$. El propósito de esta técnica es comprender el fenómeno de estudio, por lo que las limitaciones habituales de las revisiones sistemáticas (heterogeneidad de estudios, sesgos de publicación, idioma o selección, etc.) se asumen y explicitan durante el proceso de análisis.

\section{Método de búsqueda}

Pregunta PICOT: $\mathrm{P}=$ personas mayores; $\mathrm{I}=$ intervenciones apoyadas en TIC; $\mathrm{C}=$ no procede; $\mathrm{O}$ = envejecimiento saludable; $\mathrm{T}$ = no procede. Bases de datos consultadas: Cochrane Library, PubMed, EMBASE, Medline, SciELO, Medline plus y Science Direct. Se realizaron bús- quedas en lenguaje natural y en lenguaje controlado utilizando descriptores MeSH ("aging", "elderly", "informatic applications", "health services for the aged"); EMTREE ("aging”, "aged”, "elderly care", "mobile applications", "medical informatics", "information technology") y DeCS ("envejecimiento", "aplicaciones móviles", "tecnología de la información").

\section{Criterios de inclusión y exclusión}

Se seleccionaron estudios publicados en castellano e inglés en el período 2013-2018. Se incluyeron los manuscritos en función de su capacidad para explicar el fenómeno de estudio ${ }^{12}$. La exclusión de documentos se basó en criterios de saturación ${ }^{12}$. Se excluyeron publicaciones que se referían a intervenciones específicas sobre patologías o condiciones de salud tales como fragilidad, cáncer, demencias, diabetes, inmunizaciones, etc., o que incluían otros grupos de edad.

\section{Extracción de datos}

Tras la lectura íntegra de los textos, se extrajeron los datos de identificación del estudio, el tipo de diseño, el ámbito geográfico y el año de publicación.

\section{Síntesis de datos}

Se aplicó una técnica de metasíntesis cualitativa ${ }^{13}$ a través de la creación inductiva de categorías en vivo (codificación abierta), su agrupación en categorías principales (codificación axial) y la selección de los temas centrales (codificación selectiva) para la construcción del modelo.

\section{- RESULTADOS}

Se obtuvieron 262 estudios (tabla 1) y se utilizaron 22 para el análisis cualitativo (fig. 1). El 45\% de los estudios se realizaron en América del Norte, un 32\% en Europa, un 14\% en países asiáticos y un $9 \%$ en América del Sur. El 55\% eran estudios cualitativos; el 27\% revisiones sistemáticas, el $9 \%$ tenía un diseńo mixto y el $9 \%$ eran cuantitativos. La metasíntesis cualitativa se realizó en tres fases ${ }^{13}$ : una codificación abierta para identificar temas emergentes (tabla 2); una codificación axial, para agrupar estos temas en categorías (tabla 3); y, una codificación selectiva, seleccionando una categoría central, en torno a la cual organizar e integrar el resto de categorías para construir el modelo conceptual (fig. 2).

\section{- DISCUSIÓN}

El modelo conceptual desarrollado en este estudio explora la contribución de las TIC al envejecimiento saludable y aporta elementos clave para la evaluación de la efectividad de las intervenciones en este campo. En el ámbito individual, las TIC contribuyen al empoderamiento de la persona mayor. Empoderamiento en el autocuidado ${ }^{28,33}$, en la autonomía en la toma de decisiones ${ }^{33}$, en la vida independiente ${ }^{28,30}$ y en la autoeficacia en la gestión de la enfermedad y la medicación ${ }^{22,25,31}$. El empoderamiento descrito por Shearer y sus colaboradores ${ }^{36}$ como un proceso que involucra relaciones y fuerzas sociales que actúan sobre la persona y afectan su sentido de control puede ser medido a través de diversos instrumentos ${ }^{37}$. Analizado como resultado y no como intervención, el empoderamiento hace referencia al esfuerzo de ejercer 
Tabla 1. Búsquedas en bases de datos por lenguaje natural y lenguaje controlado

\begin{tabular}{|c|c|c|c|c|}
\hline BD & $\begin{array}{l}\text { Estrategias de búsqueda } \\
\text { Lenguaje natural }\end{array}$ & $\mathbf{N}^{0}$ & $\begin{array}{l}\text { Estrategias de búsqueda } \\
\text { Lenguaje controlado }\end{array}$ & $\mathrm{N}^{0}$ \\
\hline Cochrane Library & $\begin{array}{l}\text { "active aging" AND "mobile health" AND "ICT applications" AND } \\
\text { "older adults" OR "e-health" OR "health information technology" }\end{array}$ & 2 & $\begin{array}{l}\text { "aging" AND "informatics applications" OR "elderly" AND "health } \\
\text { services for the aged" }\end{array}$ & 1 \\
\hline PubMed & $\begin{array}{l}\text { "active aging" OR "older adults" OR "information and } \\
\text { communication Technologies" AND "informatics applications" }\end{array}$ & 10 & $\begin{array}{l}\text { "aging" AND "informatics applications" OR "elderly" AND "health } \\
\text { services for the aged" }\end{array}$ & 26 \\
\hline SciELO & & & $\begin{array}{l}\text { "aplicaciones móviles" AND "envejecimiento" OR "tecnología de } \\
\text { la información" }\end{array}$ & 27 \\
\hline Medline plus & $\begin{array}{l}\text { "active aging" OR "older adults" OR "information and } \\
\text { comunication Technologies" AND "app" (35) } \\
\text { "older adults" OR "health information technology" AND "app" (32) }\end{array}$ & 67 & $\begin{array}{l}\text { "aging" OR "elderly care" AND "medical informatics" AND } \\
\text { "information technology" }\end{array}$ & 19 \\
\hline
\end{tabular}



Figura 1. Esquema del estudio. 
Tabla 2. Temas emergentes extraídos de los estudios

\begin{tabular}{|c|c|c|}
\hline Autor, año, país & Objetivo & Muestra \\
\hline $\begin{array}{l}\text { Backonja U, Hall AK, } \\
\text { Thielke S. }{ }^{14} \\
\text { (2014) Estados Unidos }\end{array}$ & $\begin{array}{l}\text { Explicar la conducta de } \\
\text { las personas mayores } \\
\text { en relación con la } \\
\text { tecnología }\end{array}$ & 3 casos de estudio \\
\hline $\begin{array}{l}\text { Beard J, Officer A, Araujo } \\
\text { de Carcalho I, Sadana R, } \\
\text { Pot AM, Michel JP, et al. }{ }^{15} \\
\text { (2016) Switzerland }\end{array}$ & $\begin{array}{l}\text { Proveer un marco } \\
\text { de trabajo para la } \\
\text { acción global en } \\
\text { envejecimiento activo }\end{array}$ & Población mundial \\
\hline $\begin{array}{l}\text { Brenner S, Kaushal R, } \\
\text { Grinspan Z, Joyce C, Kim } \\
\text { I, Allard RJ, et al. }{ }^{16} \\
\text { (2015) Estados Unidos }\end{array}$ & $\begin{array}{l}\text { Identificar los efectos } \\
\text { de las TIC sobre } \\
\text { resultados en seguridad } \\
\text { en pacientes }\end{array}$ & $\begin{array}{l}4 \text { bases de datos de } \\
2001 \text { a 2012; } \\
68 \text { artículos incluidos } \\
\text { en la revisión; } \\
25 \text { estudios incluidos } \\
\text { en la síntesis cualitativa }\end{array}$ \\
\hline
\end{tabular}

Demiris G, Thompson HJ, Validar una plataforma Reeder B, Wilamowska K, que integra captura de Zaslavsky $0 .{ }^{17}$ (2016) Estados Unidos parámetros cognitivos

Fischer S, David D, Crotty BH, Dierks M, Safran Ch. ${ }^{18}$

(2014) Estados Unidos

\section{Explorar el uso y}

Gustafson D Sr, McTavish

F, Gustavson D Jr,

Mahoney RA, Lee JD,

Quanbeck A et al. ${ }^{19}$

(2015) Estados Unidos

Garavand A, Samadbeik

M, Kafashi M, Abhari

$\mathrm{Sh}^{20}$

(2017) Irán

Isakovic M, Sedlar U, Volk
M, Bester J. ${ }^{21}$
(2016) Slovenia

y adaptaciones
necesarias para el uso
de una aplicación en
población mayor

Kalem G, Turhan C.22

(2015) Turquía de datos. 361 artículos 64,2 años) parámetros funcionales, quiosco de telesalud y asesoramiento sobre

la aceptación de

la tenología de

información para

la salud entre las

personas mayores

65 participantes pertenecientes a una comunidad de personas jubiladas (78 a 94 años)

Cualitativo a través de la evaluación de la experiencia de la persona usuaria

Testear los efectos de la tecnología Elder Tree

Identificar y categorizar los factores determinantes de la adopción de sistemas de comunicación de salud móvil

Explorar la transferibilidad y adaptaciones población mayor

Analizar las aplicaciones para el manejo de la enfermedad y el bienestar a través de aplicaciones móviles
106 artículos

seleccionados en la revisión, de los que 96 son incluidos en la síntesis cualitativa

\section{0 participantes}

(personas mayores de 65 años y personas cuidadoras)

Búsqueda en 6 bases seleccionados,

9 estudios incluidos en el análisis

10 participantes mayores de 50 años (media de edad

Estudios relacionados con 10 aplicaciones móviles

Tipo de estudio

Cualitativo a través del estudio de casos

Estudio mixto; 8 bases de datos, paneles de personas expertas y revisión por pares

Revisión sistemática

y síntesis cualitativa

Beneficios variables de las TIC en salud según tipología. Los soportes de decisión clínica son las intervenciones más estudiadas. Se necesitan más estudios para evaluar los efectos de las TIC sobre la seguridad del paciente y los cuidados a largo plazo. Se requiere una mayor uniformidad en el sistema de categorización de las TIC para facilitar la comparación de resultados y más estudios longitudinales para captar su impacto

El adecuado y personalizado entrenamiento de las personas mayores y la adaptación de las aplicaciones a sus limitaciones funcionales favorecen su participación en intervenciones relacionadas con tecnologías.La informática puede dar soporte y facilitar el asesoramiento integral para el bienestar de las personas mayores. Las TIC facilitan la toma de decisiones, enlazan con las redes de cuidados y pueden reducir efectos adversos e identificar tendencias y modelos en salud

Las tecnologías para la vida asistida pueden ayudar a envejecer en

Revisión de la literatura y síntesis cualitativa casa, pero están insuficientemente evaluadas. Aspectos tales como la familiaridad, la confianza en la tecnología o la privacidad son considerados imprescindibles para facilitar el uso de las tecnologías para tomar decisiones de cuidados en salud en personas mayores

Cualitativo a través Protocolo para evaluar el efecto de las TIC en la calidad de vida de de entrevistas, cuestionario y grupos focales

Revisión sistemática las personas mayores y la reducción de costes en atención sanitaria. Sugiere que la tecnología debe ser desarrollada mediante un trabajo estrecho con la comunidad a la que va dirigida para garantizar su uso y los resultados esperados

La salud móvil tiene un importante papel en la provisión de servicios de cuidados de salud. La facilidad percibida de uso y la utilidad percibida son los factores más importantes que afectan a la adopción de un sistema de comunicación de salud móvil

Cualitativo a través de test de usabilidad y cuestionarios

Revisión de la literatura
Adaptaciones en tamaño, visibilidad y comprensibilidad de botones y símbolos. Uso de tips de ayuda y explicaciones adicionales. Contraste de color necesario para personas con pérdida de la capacidad visual. Uso de fondos de colores fuertes para mejorar la legibilidad de los textos

Las aplicaciones para móviles se dirigen al manejo de enfermedades (asma, trastornos psicológicos, diabetes, dolor de cabeza, etc.). Las personas mejoran su autoeficacia y aportan más información a profesionales, lo que contribuye a su recuperación y bienestar 
Tabla 2. Temas emergentes extraídos de los estudios (cont.)

\begin{tabular}{|l|l|}
\multicolumn{1}{|c|}{ Autor, año, país } & \multicolumn{1}{c|}{ Objetivo } \\
\hline $\begin{array}{l}\text { Kim JY, Lee KH, Kim SH, } \\
\text { Kim KH, Kim JH, Han JS, } \\
\text { et al. }{ }^{23} \\
\text { (2013) Corea }\end{array}$ & $\begin{array}{l}\text { Desarrollar y pilotar un } \\
\text { programa de servicios } \\
\text { de mensajería de textos } \\
\text { personalizados para el } \\
\text { control del peso }\end{array}$ \\
\hline $\begin{array}{l}\text { LeRouge C, Ma J, Sneha S, } \\
\text { Tolle K.24 } \\
\text { (2013) Estados Unidos }\end{array}$ & $\begin{array}{l}\text { Explorar el diseño } \\
\text { centrado en el usuario, } \\
\text { como herramienta } \\
\text { metodológica para el } \\
\text { diseño y desarrollo de } \\
\text { tecnologías de salud } \\
\text { para población }\end{array}$ \\
\hline
\end{tabular}

Mira JJ, Navarro I, Botella
F, Borrás F, Nuño-Solinís R, Orozco D, et al. ${ }^{25}$ (2014) España

Rigby M, Ronchi E, Graham S. ${ }^{26}$

(2013) Estados Unidos

Diseñar, implementar y evaluar una aplicación de automanejo de medicación

Diseñar estrategias para el futuro de los sistemas sanitarios y el bienestar

\begin{tabular}{l}
\multicolumn{1}{c}{ Muestra } \\
340 participantes \\
con IMC superior a \\
24 para el desarrollo \\
del programa y 102 \\
participantes para el \\
pilotaje
\end{tabular}

54 participantes en

9 grupos focales

30 entrevistas semiestructuradas de la población

\section{Rivas-Nieto A, Málaga G, Determinar el uso Ruiz-Grosso P, Huayanay- de TIC en pacientes Espinoza CA, Curioso WH. ${ }^{27}$ con dislipemia, HTAy (2015) Perú diabetes. Explorar la adherencia al tratamiento y su percepción sobre el uso de TIC}

Rodríguez Gómez R. ${ }^{28}$ (2017) Colombia

Analizar la trascendencia de conceptos clave para entender el trasfondo de la salud móvil

\section{3 pacientes $y$ 7 profesionales en el estudio cualitativo 99 participantes en el experimental}

\section{5 participantes}

Tाpo de estudio

Cuantitativo a

través de un

cuestionario

autoadministrado

y medidas antropométricas

\section{Cualitativo.}

Investigaciónacción. Observación participante en hospitales; 9 grupos focales y 30 entrevistas con profesionales sanitarios

Estudio mixto Grupos nominales, grupo focal y estudio experimental con grupo de control

Cualitativo a través de paneles de personas expertas

\section{6 participantes} (mayores de 18 años) con al menos uno de los diagnósticos considerados: dislipemia, diabetes, HTA

Se identificaron

90 estudios en la búsqueda de los que se seleccionaron 46
Cuantitativo. Estudio observacional descriptivo a través de encuesta validada por personas expertas

Revisión de la literatura

Aspectos clave en el diseño: facilidad de uso, tamaño de letra grande; incluir fotografías y alertas. La «app» mejora la adherencia al tratamiento, ayuda a recordar la medicación, contribuye a reducir errores sistemáticos y aumenta la percepción de independencia en el manejo de su medicación

Cambios demográficos y la presión externa para modernización y eficiencia del sistema sanitario. Las redes sociales representan un nuevo paradigma de participación en el cuidado. El éxito depende del compromiso y la implicación de las personas en el diseño. Las TIC deben estar basadas en la evidencia

La mayoría de pacientes están dispuestos a recibir recordatorios y a ser parte de programas de seguimiento usando móviles e Internet. Los mensajes de texto o Internet pueden ser útiles para el desarrollo de programas de salud, al contar con la aceptación de pacientes y cuidadoras, quienes podrían ser agentes clave en el desarrollo e implementación de estos programas

Conceptos clave: cutura, ciberespacio, inteligencia colectiva, acción, interacción, Internet, salud móvil y «apps». Hace falta conocer y entender la cibercultura para lograr su aplicación práctica. La noción de interacción es primordial. La salud móvil constituye una tendencia hacia la democratización de la salud. Empoderar a las personas para el autocuidado a través de herramientas digitales representa una revolución

\begin{tabular}{ll} 
Sevilla M, Salgado M, & Dar a conocer el impacto \\
Osuna N.29 & que tiene el uso de \\
(2015) México & $\begin{array}{l}\text { ordenadores en la vida } \\
\text { cotidiana de las personas } \\
\text { mayores }\end{array}$ \\
\hline
\end{tabular}

Stav E, Walderhaug $S$, Mikalsen M, Hanke S, Benc 1.30 (2013) Noruega

Tseng MH, Wu HC. ${ }^{31}$
(2014) Taiwan

Evaluar el diseño y desarrollo de una plataforma para construir servicios asistidos para personas mayores

Evaluar el diseño y la demostración de un sistema de soporte para la medicación segura

\section{0 personas mayores}

Cualitativo a través de un estudio de casos seleccionados mediante un muestreo propositivo

\section{3 participantes} (familiares, profesionales expertos en demencia, pacientes y proveedores de cuidados)

20 participantes

Cualitativo a través de técnicas de evaluación de la experiencia de personas usuarias

\section{Cualitativo a} través de test de experiencia de personas usuarias con cuestionario
Promoción del envejecimiento saludable implica socialización, prevención y control de enfermedades crónicas, mantenimiento de la funcionalidad y mejora de la autoestima, la calidad de vida y el bienestar. Las personas mayores se sienten motivadas y seguras en el desempeño de actividades relacionadas con las TIC

Importante la simplicidad en la interfaz para que las personas mayores utilicen las soluciones TIC. Los sistemas de vida asistida requieren de una plataforma basada en una arquitectura orientada a servicios para cubrir las necesidades de las personas mayores usuarias

La percepción de utilidad, facilidad de uso, confianza e intención de uso de una aplicación móvil para el control de la medicación es alta. La privacidad y la seguridad son elementos críticos que favorecen la utilización de las aplicaciones móviles 
Tabla 2. Temas emergentes extraídos de los estudios (cont.)

\begin{tabular}{|c|c|c|c|c|}
\hline Autor, año, país & Objetivo & Muestra & Tाpo de estudio & Temas emergentes \\
\hline $\begin{array}{l}\text { Vedel I, Akhlaghpour S, } \\
\text { Vaghefi I, Bergman H, } \\
\text { Lapointe L.32 } \\
\text { (2013) Canadá }\end{array}$ & $\begin{array}{l}\text { Proporcionar } \\
\text { una tipología de } \\
\text { aplicaciones TIC. } \\
\text { Determinar los } \\
\text { resultados y factores } \\
\text { asociados al uso }\end{array}$ & $\begin{array}{l}112 \text { estudios incluidos } \\
\text { en la revisión }\end{array}$ & $\begin{array}{l}\text { Revisión } \\
\text { sistemática de la } \\
\text { literatura }\end{array}$ & $\begin{array}{l}\text { Tipología: telecuidados, registro electrónico de salud, sistemas } \\
\text { de soporte de decisiones, sitios web para pacientes o cuidadoras } \\
\text { familiares y tecnologías de información para la asistencia. Para } \\
\text { maximizar el éxito en la implementación se debe integrar su uso } \\
\text { en el flujo de trabajo y rutina de profesionales. La educación y } \\
\text { entrenamiento de las personas usuarias es crucial }\end{array}$ \\
\hline $\begin{array}{l}\text { Vollenbroek-Hutten M, } \\
\text { Jansen-Kosterink S, } \\
\text { Tabak M, Feletti L, Zia G, } \\
\text { N'dja A, et al. }{ }^{33} \\
\text { (2017) Países Bajos }\end{array}$ & $\begin{array}{l}\text { Descubrir el campo de } \\
\text { posible de aplicación de } \\
\text { las TIC para el soporte } \\
\text { al manejo clínico de las } \\
\text { personas mayores }\end{array}$ & $\begin{array}{l}673 \text { pacientes y } \\
104 \text { participantes en } \\
\text { plataforma multimodal } \\
\text { de servicios }\end{array}$ & $\begin{array}{l}\text { Cualitativo a través } \\
\text { del análisis de } \\
2 \text { casos de TIC } \\
\text { para el soporte } \\
\text { del manejo clínico } \\
\text { de enfermedades } \\
\text { crónicas }\end{array}$ & $\begin{array}{l}\text { Las TIC ofrecen alternativas para su implementación en la práctica } \\
\text { diaria promoviendo la autonomía en el autocuidado. } \\
\text { La actitud de profesionales hacia la telemedicina influye en la } \\
\text { percepción y adherencia en el uso de las tecnologías por parte de } \\
\text { las personas mayores }\end{array}$ \\
\hline $\begin{array}{l}\text { Wildenbos G, Peute L, } \\
\text { Jaspers MA. }{ }^{34} \\
\text { (2015) Países Bajos }\end{array}$ & $\begin{array}{l}\text { Desarrollar un modelo } \\
\text { conceptual a partir } \\
\text { de los impulsos } \\
\text { motivacionales, físicos } \\
\text { y visuales. Analizar los } \\
\text { aspectos de usabilidad } \\
\text { de una «app» }\end{array}$ & 5 evaluadores & $\begin{array}{l}\text { Cualitativo a } \\
\text { través de un panel } \\
\text { virtual de personas } \\
\text { expertas }\end{array}$ & $\begin{array}{l}\text { Basar el desarrollo de aplicaciones en la comprensión de las } \\
\text { barreras que afectan a la usabilidad. El modelo conceptual } \\
\text { desarrollado provee un modelo de referencia para evaluar y analizar } \\
\text { los problemas de usabilidad desde la perspectiva de este grupo de } \\
\text { población }\end{array}$ \\
\hline $\begin{array}{l}\text { Williamson SS, Goman } \\
\text { PN, Jimison HB. }{ }^{35} \\
\text { (2014) EE.UU. }\end{array}$ & $\begin{array}{l}\text { Medir los beneficios } \\
\text { percibidos y las } \\
\text { barreras de } 12 \\
\text { tecnologías para el } \\
\text { cuidado de mayores por } \\
\text { parte de cuidadores a } \\
\text { larga distancia }\end{array}$ & 10 participantes & $\begin{array}{l}\text { Cualitativo a través } \\
\text { de entrevistas } \\
\text { virtuales } \\
\text { semiestructuradas } \\
\text { (Skype) }\end{array}$ & $\begin{array}{l}\text { Funciones que responden a las necesidades de las personas } \\
\text { cuidadoras: videoconferencia, calendarización, registro de } \\
\text { medicación, compartir fotos, registro de salud cognitiva, registro de } \\
\text { sueño, de ejercicio físico, acceso a informes médicos, recurso de } \\
\text { salud on-line y comunicación asíncrona, entre otras }\end{array}$ \\
\hline
\end{tabular}

HTA: hipertensión arterial; TIC: tecnologías de la información y de la comunicación.

Tabla 3. Categorías principales según los estudios seleccionados


$\mathbf{x}$


\begin{tabular}{|l|}
\hline Fenómeno \\
\hline Prioridades del sistema sanitario \\
\hline Prioridades de las personas \\
\hline Perfiles de personas usuarias \\
\hline
\end{tabular}

\begin{tabular}{l}
\hline Perfiles de personas usuarias \\
\hline Tipo de tecnologías \\
\hline Funciones \\
\hline
\end{tabular}
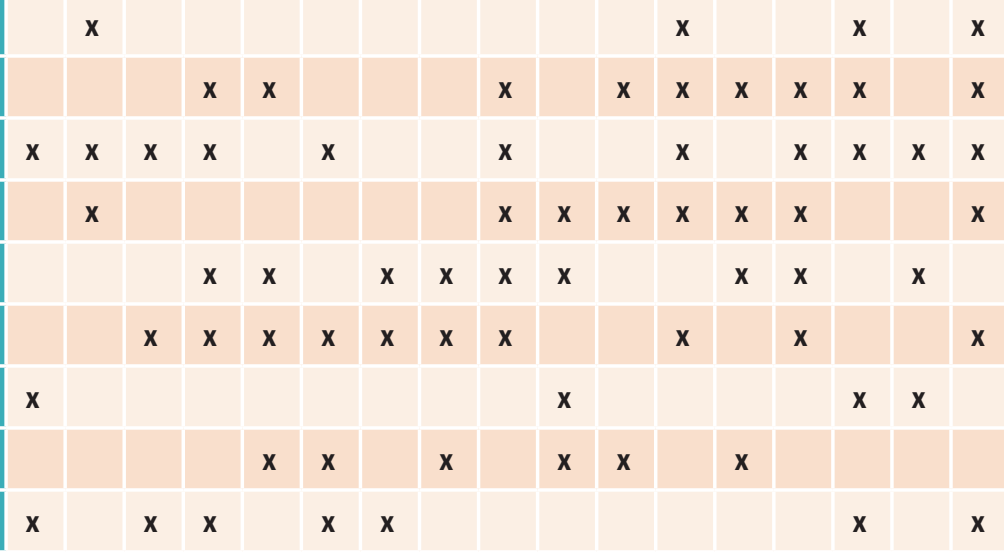

\begin{tabular}{l} 
Requisitos \\
\hline Claves de éxito
\end{tabular}






Figura 2. Modelo conceptual sobre tecnologías y envejecimiento saludable.

control e influencia sobre las decisiones que afectan a la vida de cada persona. Y es en este sentido que las TIC actúan como una herramienta que proporciona información y recursos útiles, de forma que la persona pueda entender mejor lo que le ocurre y sea capaz de manejar las situaciones por sí misma o por medio de otras personas de su red de apoyo. En el ámbito social, las TIC pueden favorecer el acceso a recursos que pueden proporcionar el refuerzo necesario para que la persona pueda tomar decisiones de forma más autónoma ${ }^{38}$.
Y es que la llamada salud móvil se convierte en una oportunidad para la gestión de las condiciones de salud ${ }^{28}$, al tiempo que supone toda una revolución en el autocuidado. En esta idea redundan otros autores que señalan cómo las tecnologías de información de salud tienen un gran potencial para el empoderamiento de las personas mayores a la hora de tomar un rol proactivo en su salud ${ }^{24}$. Por otra parte, los desarrollos informáticos de soporte al bienestar que presentan una visión holística de las necesidades de las personas mayores 
facilitan la toma de decisiones, enlazan con las redes informales de cuidados y pueden reducir eventos adversos de salud ${ }^{17}$.

Para conseguir este empoderamiento, la tecnología debe ser desarrollada mediante un trabajo estrecho con la comunidad a la que va dirigida $^{19}$, tener en cuenta las posibles barreras asociadas al envejecimiento, tales como la falta de familiaridad y de confianza en las propias tecnologías $^{18}$ y las necesidades especiales de la población mayor ${ }^{22}$, en particular las asociadas al deterioro funcional y cognitivo ${ }^{34}$.

El empleo de las TIC dirigido hacia las personas mayores debe tener muy en cuenta las características de esta población; además de la facilidad y la intuición en los productos, se les exige que sean veraces y precisos a fin de conseguir su adherencia. Es aquí donde surge la necesidad de investigar el diseńo centrado en la persona ${ }^{22}$ en cuanto al análisis de sus necesidades, deseos y limitaciones, como herramienta metodológica que informe el desarrollo de tecnologías de salud para la población mayor. La simplicidad en la interfaz ${ }^{30}$, la facilidad de uso ${ }^{25}$, la educación y el entrenamiento en su utilización ${ }^{22,32}$, la forma cómo se implementan las TIC en la práctica diaria ${ }^{33}$, la facilidad percibida de uso, la utilidad percibida y las condiciones facilitadoras ${ }^{20} \mathrm{y}$ la comprensibilidad ${ }^{21}$, entre otros, son factores que afectan a la adopción de un sistema de tecnología de información de salud.

Así, por ejemplo, los mensajes de promoción de la salud a través de la mensajería de texto tienen un alto índice de satisfacción cuando van dirigidos a problemas específicos sentidos como tales por la población destinataria ${ }^{23}$. Además, pueden ser útiles para el desarrollo de programas de salud, al contar con la aceptación de pacientes y personas cuidadoras ${ }^{27}$.

En el ámbito comunitario, el modelo entiende que las TIC favorecen la participación de las personas mayores en los sistemas de cuidados y promueven la democratización de la salud ${ }^{6,15,26}$. La participación ciudadana se hace efectiva en la orientación de los servicios a las necesidades de las personas mayores; en la sostenibilidad del sistema de cuidados; en la generación de entornos amigables, y en la generación de nuevos conocimientos ${ }^{15}$. En este campo, el papel de las TIC es fundamental. El registro y análisis permanente de la información respecto a las condiciones de salud y los hábitos de vida contribuye a la personalización de los servicios y favorece el cuidado de la salud de esta población.

Otro de los resultados clave que se desprende del modelo es la necesidad de diseñar instrumentos de evaluación flexibles que se adapten a la diversidad de TIC en función de su tipología, funciones y requisitos. Los sistemas de telecuidado, los registros electrónicos de salud, los sistemas de soporte de decisiones, los sitios web para personas mayores y cuidadoras, las tecnologías de información para la vida asistida ${ }^{32}$, sumadas a las aplicaciones para móviles dirigidas al manejo de patologías tan diversas como el asma, los trastornos psicológicos, la diabetes, las migrańas o la actividad física, etc. ${ }^{22}$, abren un enorme campo de investigación que aporte información útil para comprender y evaluar cómo la tecnología puede servir al propósito del bienestar individual y el desarrollo social y económico de las sociedades altamente envejecidas $^{14,29,35}$.

En conclusión, las TIC contribuyen al empoderamiento de las personas para el autocuidado; mejoran su autoeficacia, promueven su autonomía en la toma de decisiones; favorecen su participación efectiva en los sistemas sanitarios y de cuidados; y contribuyen a democratizar la salud. Su utilización depende de la participación de las personas mayores en su diseño y su adaptación a los entornos y preferencias de las personas usuarias, su simplicidad, comprensibilidad, utilidad percibida y facilidad de uso.

\section{Conflicto de intereses}

Los autores y autoras del presente manuscrito declaramos que no existe ningún conflicto de interés que pueda sesgar el trabajo presentado.

\section{Agradecimientos}

Queremos expresar nuestro agradecimiento a María del Pilar Toro Sánchez-Blanco y a Carlos Bruquetas Callejo por su inestimable colaboración durante el desarrollo del estudio.

\section{- BIBLIOGRAFÍA}

1. Kalache A. Envejecimiento Activo. Un marco político ante la revolución de la longevidad. ILC-Brasil. Granada: Escuela Andaluza de Salud Pública; 2015.

2. Whittington FJ. Form ageism to the longevity revolution: Robert Butler, Pioneer. The Gerontologist. 2014;54(6):1064-74.

3. UNFPA, Help Age International. Ageing in the Twenty-First Century: a celebration and a challenge. New York/London: UNPFA/ Help Age International; 2012

4. Zunzunegui MV. Evolución de la discapacidad y la dependencia. Una mirada internacional. Gac Sanit. 2011;25(S):15-20.

5. Kalache A. Coping with the Longevity Revolution. Cien Saude Colet 2014;19(8):3306.

6. Pittiani M. Papel de las TIC en el envejecimiento. Lychnos. 2012;8:60-4.

7. Groves P, Kayyali B, Knott D, Van Kuiken S. The "big data" revolution in healthcare. Accelerating value and innovation. New York: McKinsey \& Company; 2013

8. Plaza I, Martín L, Martin S, Medrano C. Mobile applications in an aging society: Status and trends. The Journal of Systems and Software. 2011;84(11):1977-88.

9. Agudo S, Pascual MA, Fombona J. Usos de las herramientas digitales entre las personas mayores. Comunicar. 2012;39(29):193-201.

10. Kowal P, Chatterii S, Naidoo N, Biritwum R, Fan W, Lopez Ridaura R, et al, and the SAGE Collaborators. Data resource profile: The World Health Organization Study on Global Ageing and Adult Health (SAGE). Int J Epidemiol. 2012;41:1639-49.

11. Carreño SP, Chaparro L. Metasíntesis: discusión de un abordaje metodológico. Ciencia y Enfermería. 2015:21(3):123-31.

12. Gálvez A. Métodos contemporáneos de síntesis científica: una propuesta de homogeneidad. Med Clin (Barc). 2003;121(7):256-63.
13. Carrillo GM, Gómez 0J, Vargas E. Metodologías en metasíntesis Ciencia y Enfermería. 2008;14(2):13-9.

14. Backonja U. Hall AK, Thielke S. Older adults' current and potentia uses of information technologies in a changing world. A theoretica perspective. Int J Aging Hum Dev. 2014;80(1):41-63.

15. Beard JR, Officer A, Araujo de Carvalho I, Sadana R, Pot AM, Michel JP. et al. The World report on ageing and health: a policy framework for healthy ageing. The Lancet. 2016;387(21):2145-54

16. Brenner S, Kaushal R, Grinspan Z, Joyce C, Kim I, Allard R, et al. Effects of health information technology on patient outcomes: a systematic review. J Am Med Inform Assn. 2015;23(5):1016-36.

17. Demiris G, Thompson H, Reeder B, Wilamowska K, Zaslavsky 0 . Using informatics to capture older adults' wellness. Int J Med Inform. 2013:82(11):e232-41

18. Fischer S, David D, Crotty B, Dierks M, Safran C. Acceptance and use of health information technology by community-dwelling elders. Int J Med Inform. 2014:83(9):624-35.

19. Gustafson D, McTavish F, Gustafson D, Mahoney J, Johnson R, Lee $J$, et al. The effect of an information and communication technology (ICT) on older adults' quality of life: study protocol for a randomized control trial. Trials. 2015;16(1)

20. Garavand A, Samadbeik M, Kafashi M, Abhari S. Acceptance of Health Information Technologies, Acceptance of Mobile Health: A Review Article. J Biomed Phys Eng. 2017:7(4):403-8.

21. Isakovi'c M, Sedlar U, Volk M, Bešter J. Usability Pitfalls of Diabetes mHealth Apps for the Elderly. J Diabetes Res. 2016;2016:1-9.

22. Kalem G. Turhan C. Mobile Technology Applications in the Healthcare Industry for Disease Management and Wellness. Procedia Social and Behavioral Sciences. 2015;195:2014-2018.
23. Kim J, Lee K, Kim S, Kim K, Kim J, Han J, et al. Needs analysis and development of a tailored mobile message program linked with electronic health records for weight reduction. Int J Med Inform. 2013;82(11):1123-32

24. LeRouge C, Ma J, Sneha S, Tolle K. User profiles and personas in the design and development of consumer health technologies. Int $J$ Med Inform. 2013:82(11):e251-e268.

25. Mira J, Navarro I, Botella F, Borrás F, Nuño-Solinís R, Orozco D, et al. A Spanish Pillbox App for Elderly Patients Taking Multiple Medications: Randomized Controlled Trial. J Med Internet Res. 2014;16(4):e99.

26. Rigby M, Ronchi E, Graham S. Evidence for building a smarte health and wellness future-Key messages and collected visions from a Joint OECD and NSF workshop. Int J Med Inform. 2013:82(4):209-19

27. Rivas-Nieto A, Málaga G, Ruiz-Grosso P, Huayanay-Espinoza C, Curioso W. Uso y percepciones de las tecnologías de información y comunicación en pacientes con hipertensión arterial, dislipidemia 0 diabetes de un hospital nacional de Lima, Perú. Rev Peru Med Exp Salud Pública. 2015;32(2):283

28. Rodríguez Gómez R. Cibercultura, internet y salud móvil. Universidad y Salud. 2017:19(2):293.

29. Sevilla Caro M, Salgado Soto M, Osuna Millán N. Envejecimiento activo. Las TIC en la vida del adulto mavor. Revista Iberoamericana para la Investigación y el Desarrollo Educativo. 2015;6(11):574.

30. Stav E, Walderhaug S, Mikalsen M, Hanke S, Benc I. Development and evaluation of SOA-based AAL services in real-life environments: A case study and lessons learned. International Journal of Medical Informatics. 2013;82(11):e269-93. 
Mónica Padial Espinosa, Sandra Pinzón Pulido, Juan Manuel Espinosa Almendro, Alexandre Kalache y Francisco Garrido Peña

Longevidad y revolución digital. Contribución de las tecnologías de la información y de la comunicación al envejecimiento saludable

31. Tseng MH, Wu HC. A cloud medication safety support system using QR code and Web services for elderly outpatients. Technol Health Care. 2014;22(1):99-113.

32. Vedel I, Akhlaghpour S, Vaghefi I, Bergman H, Lapointe L. Health information technologies in geriatrics and gerontology: a mixed systematic review. J Am Med Inform Assn. 2013:20(6):1109-19.

33. Vollenbroek-Hutten M, Jansen-Kosterink S, Tabak M, Feletti L, Zia G, N'dja A, et al. Possibilities of ICT-supported services in the clinical management of older adults. Aging Clin Exp Res. 2017;29(1):49-57.

34. Wildenbos G, Peute L, Jaspers M. A framework for evaluating mHealth tools for Older Patients on Usability. Stud Health Techno Inform. 2015;210:783-7.

35. Williamson SS, Gorman PN, Jimison HB. A mobile/web App for long distance caregivers of older adults: functional requirements and design implications from a user centered design process. AMIA Annu Symp Proc. eCollection 2014;1960-9.
36. Shearer NBC, Fleury J, Ward KZ, O'Brien AM. Empowerment interventions for older adults. Western J Nurs Res. 2012;34(I):24-51.

37. Bakker L, Van Brakel WH. Empowerment assessment tools in people with disabilities in developing countries. A systematic literature review. Lepr Rev. 2012;83:129-53.

38. Quintero MV, Fiqueroa N, García F, Suárez M. Educación sanitaria para la calidad de vida y empoderamiento de la salud de personas mayores. Gerokomos. 2017;28(1):9-14. 